\title{
Finding minimum clique capacity
}

\author{
Maria Chudnovsky ${ }^{1}$ \\ Columbia University, New York, NY 10027 \\ Sang-Il Oum ${ }^{2}$ \\ KAIST, Daejeon, Republic of Korea \\ Paul Seymour ${ }^{3}$ \\ Princeton University, Princeton, NJ 08544
}

July 21, 2009; revised July 21, 2009

${ }^{1}$ Supported by NSF grant DMS-0758364.

${ }^{2}$ Supported by

${ }^{3}$ Supported by ONR grant N00014-01-1-0608. 


\begin{abstract}
Let $C$ be a clique of a graph $G$. The capacity of $C$ is defined to be $(|V(G) \backslash C|+|D|) / 2$, where $D$ is the set of vertices in $V(G) \backslash C$ that have both a neighbour and a non-neighbour in $C$. We give a polynomial-time algorithm to find the minimum clique capacity in a graph $G$. This problem arose as an open question in a study [1] of packing vertex-disjoint induced three-vertex paths in a graph with no stable set of size three.
\end{abstract}




\section{Introduction}

In this paper, all graphs are finite and have no loops or multiple edges. A clique is a subset of $V(G)$ of vertices that are pairwise adjacent. A subset $X$ of $V(G)$ is stable if all members of $X$ are pairwise nonadjacent, and $\alpha(G)$ denotes the cardinality of the largest stable subset of $V(G)$. If $C \subseteq V(G)$, a vertex $v \in V(G) \backslash C$ is complete to $C$ if $v$ is adjacent to every member of $C$, and anticomplete to $C$ if it has no neighbour in $C$.

Let $C$ be a clique of a graph $G$. Let $A, B, D \subseteq V(G) \backslash C$ be respectively the sets of all vertices $v \in V(G) \backslash C$ such that

- $v$ is complete to $C$

- $v$ is anticomplete to $C$

- $v$ has both a neighbour and a non-neighbour in $C$.

Thus $A \cup B \cup D=V(G) \backslash C$, and if $C \neq \emptyset$ then $A, B, D$ are pairwise disjoint.

The problem of choosing $C$ with $|C|$ maximum is NP-hard. On the other hand, it is easy to find a clique $C$ with $|C|+|A| / 2$ maximum in polynomial time. (To see this, take two copies $p(v), q(v)$ of each vertex $v$ of $G$, and for distinct $u, v \in V(G)$, make $p(u), q(v)$ adjacent if $u, v$ are nonadjacent in $G$, forming a bipartite graph $H$. Find the maximum stable set $X$ in $H$, and let $C$ be the set of all $v \in V(G)$ such that $p(v), q(v)$ are both in $X$. It is easy to check that $C$ is the clique of $G$ with $|C|+|A| / 2$ maximum.) We define the capacity cap $(C)$ of the clique $C$ to be $(|A|+|B|) / 2+|D|$, and in this paper we study finding a clique $C$ with minimum capacity (that is, with $|C|+(|A|+|B|) / 2$ maximum). It turns out that we can modify the simple algorithm just given to solve the capacity problem.

A seagull in $G$ is an induced three-vertex path in $G$. In [1] the problem of packing vertex-disjoint seagulls was studied, and a min-max formula was given for the maximum seagull packing in graphs with $\alpha(G) \leq 2$, the following (an antimatching means a matching in the complement graph, and the five-wheel is the graph with six vertices in which one vertex is complete to the vertex set of a cycle of length five):

1.1 Let $G$ be a graph with $\alpha(G) \leq 2$, and let $k \geq 0$ be an integer, such that if $k=2$ then $G$ is not a five-wheel. Then $G$ has $k$ pairwise disjoint seagulls if and only if

- $|V(G)| \geq 3 k$

- $G$ is k-connected,

- every clique of $G$ has capacity at least $k$, and

- $G$ admits an antimatching of cardinality $k$.

This did not directly yield a polynomial-time algorithm to compute the size of the optimum seagull packing, however, because we did not know how to compute in polynomial time whether every clique has capacity at least $k$, and we had to resort to the ellipsoid method. In this paper we give a polynomial-time algorithm for the missing step. We show 
1.2 There is an algorithm, with running time $O\left(n^{3.5}\right)$, which with input an $n$-vertex graph $G$, finds a clique $C$ in $G$ with minimum capacity.

We begin with the following; then 1.2 follows by running 1.3 for every vertex $c$ in turn.

1.3 There is an algorithm, with running time $O\left(n^{2.5}\right)$, which with input an n-vertex graph $G$ and a vertex $c \in V(G)$, outputs a clique $C$ containing $c$, with cap $(C)$ minimum over all cliques that contain $c$.

Proof. Here is the algorithm. Let $N$ be the set of neighbours of $c$ and $M$ the set of vertices different from $c$ that are nonadjacent to $C$. Take two copies $p(v), q(v)$ of each vertex $v \in V(G)$, and make a graph $H$ with vertex set

$$
\{p(v): v \in N\} \cup\{q(v): v \in N \cup M\}
$$

with edges as follows:

- $\{p(v): v \in N\}$ and $\{q(v): v \in N \cup M\}$ are stable sets

- for all distinct $u, v \in N, p(u)$ and $q(v)$ are adjacent if and only if $u, v$ are nonadjacent in $G$

- for all $u \in N$ and $v \in M, p(u)$ and $q(v)$ are adjacent in $H$ if and only if $u, v$ are adjacent in $G$

- for all $u \in N, p(u)$ and $q(u)$ are nonadjacent in $H$.

Thus $H$ is bipartite. Find the maximum stable subset $X$ of $V(H)$. (This takes time $O\left(n^{2.5}\right)$.) Then output

$$
\{c\} \cup\{v \in N: p(v) \in X \text { and } q(v) \in X\} .
$$

That completes the description of the algorithm. The running time is $O\left(n^{2.5}\right)$, using the algorithm of Hopcroft and Karp [2]; now we discuss its correctness. Let $X$ be the stable set of $H$ chosen by the algorithm.

(1) Let $k$ be minimum such that some clique containing c has capacity $k / 2$. Then $|X| \geq 2 n-k-2$.

For let $C$ be a clique of $G$ with $c \in C$ and $\operatorname{cap}(C)=k / 2$. Let $A, B, D$ be as usual. Thus $A, C \backslash\{c\} \subseteq N$ and $B \subseteq M$. The set

$$
\{p(v): v \in C \backslash\{c\}\} \cup\{q(v): v \in A \cup B \cup(C \backslash\{c\})\}
$$

is a stable set of $H$, with cardinality

$$
|A|+|B|+2|C|-2=2(|A|+|B|+|C|+|D|)-2 \operatorname{cap}(C)-2=2 n-k-2 .
$$

Since $X$ is a maximum stable set of $H$, it follows that $|X| \geq 2 n-k-2$. This proves (1).

Let $C=\{c\} \cup\{v \in N: p(v), q(v) \in X\}$. Thus $C$ is the set returned by the algorithm, and $C \subseteq\{c\} \cup N$. Moreover, if $u, v \in C \backslash\{c\}$ are distinct then $p(u), q(v) \in X$, and since $X$ is stable in $H$, we deduce that $p(u), q(v)$ are nonadjacent in $H$, and so $u, v$ are adjacent in $G$. Consequently $C$ is a clique of $G$. 
(2) $\operatorname{cap}(C) \leq k / 2$.

For let

$$
A=\{v \in N \backslash C: p(v) \in X \text { or } q(v) \in X\},
$$

$B=\{v \in M: q(v) \in X\}$, and $D=V(G) \backslash(A \cup B \cup C)$. Thus $|X|=2(|C|-1)+|A|+|B|$, and since $|X| \geq 2 n-k-2$ it follows that $2(|C|-1)+|A|+|B| \geq 2 n-k-2$, that is,

$$
2|C|+|A|+|B| \geq 2(|A|+|B|+|C|+|D|)-k .
$$

Consequently $|A|+|B|+2|D| \leq k$. Now since $X$ is stable in $H$, we deduce that for all $u \in C \backslash\{c\}$ and $v \in B, p(u), q(v)$ are nonadjacent in $H$, and so $u, v$ are nonadjacent in $G$. Since $B \subseteq M$, it follows that every vertex in $B$ is anticomplete to $C$. We claim that every vertex in $A$ is complete to $C$. For let $u \in C \backslash\{c\}$ and $v \in A$. Then $v \in N \backslash C$, and one of $p(v), q(v) \in X$; and so since $p(u), q(u) \in X$ and $X$ is stable in $H$, it follows that either $p(u), q(v)$ are nonadjacent in $H$ (if $q(v) \in X$ ) or $q(u), p(v$ ) are nonadjacent in $H$ (if $p(v) \in X$ ). In either case it follows that $u, v$ are adjacent in $G$, and so $v$ is complete to $C$, as claimed. Consequently $\operatorname{cap}(C) \leq|A|+|B|+2|D| \leq k / 2$. This proves (2).

From (2), and the choice of $k$, it follows that $\operatorname{cap}(C)=k / 2$, and so the clique returned by the algorithm is indeed a clique containing $c$ with minimum capacity. This proves 1.3.

\section{References}

[1] Maria Chudnovsky and Paul Seymour, "Packing seagulls", submitted for publication.

[2] J. E. Hopcroft and R. M. Karp, "An $n^{5 / 2}$ algorithm for maximum matchings in bipartite graphs", SIAM Journal on Computing 2 (1973), 225-231. 